\title{
Chapter 1 \\ Numerical Analysis of Optimality-System POD for Constrained Optimal Control
}

\author{
Eva Grimm, Martin Gubisch, and Stefan Volkwein *
}

\begin{abstract}
In this work linear-quadratic optimal control problems for parabolic equations with control and state constraints are considered. Utilizing a Lavrentiev regularization we obtain a linear-quadratic optimal control problem with mixed controlstate constraints. For the numerical solution a Galerkin discretization is applied utilizing proper orthogonal decomposition (POD). Based on a perturbation method it is determined by a-posteriori error analysis how far the suboptimal control, computed on the basis of the POD method, is from the (unknown) exact one. POD basis updates are computed by optimality-system POD. Numerical examples illustrate the theoretical results for control and state constrained optimal control problems.
\end{abstract}

\subsection{Introduction}

In this paper we consider a certain class of linear-quadratic optimal control problems governed by linear evolution equations together with control and state constraints. Such linear-quadratic problems are especially interesting as they occur for example as subproblems in each step of sequential quadratic programming (SQP) methods for solving nonlinear problems. For the numerical solution we apply a Galerkin approximation, which is based on proper orthogonal decomposition (POD), a method for deriving reduced-order models of dynamical systems; see [7, 11, 19], for instance. In order to ensure that the POD suboptimal solutions are sufficiently accurate, we derive an a-posteriori error estimate for the difference between the exact (unknown) optimal control and its suboptimal POD approximations. The proof relies on a perturbation argument [5] and extends the results of [8, 22, 25].

S. Volkwein

University of Konstanz, Department of Mathematics and Statistics, Universitätsstr. 10, 78457 Konstanz, Germany, e-mail: Stefan.Volkwein@uni-konstanz.de

* This work was supported by the DFG project A-Posteriori-POD Error Estimators for Nonlinear Optimal Control Problems governed by Partial Differential Equations, grant VO 1658/2-1. 
However, to obtain the state data underlying the POD reduced order model, it is necessary to solve once the full state system and consequently the POD approximations depend on the chosen parameters for this solve. To be more precise, the choice of an initial control turned out to be essential. When using an arbitrary control, the obtained accuracy was not at all satisfying even when using a huge number of basis functions whereas an optimal POD basis (computed from the FE optimally controlled state) led to far better results. To overcome this problem different techniques for improving the POD basis have been proposed. Here, we will apply the so called optimality system POD (OS-POD) introduced in [17]. The idea of OS-POD is straightforward: include the equations determining the POD basis in the optimization process. A thereby obtained basis would be optimal for the considered problem. We follow the ideas in $[6,26]$, where OS-POD is combined efficiently with an aposteriori error estimation to compute a better initializing control. The POD basis is then determined from this control and the a-posteriori error estimate ensures that the optimal control problem is solved up to a desired accuracy. Let us refer to [1] where the trust-region POD method is introduced as a different update strategy for the POD basis.

The paper is organized in the following manner: In Section 1.2 we introduce our optimal control problem with control and state constraints. To deal numerically with the state constraints a Lavrentiev regularization is utilized in Section 1.3. The POD method is explaine briefly in Section 1.4. In Section 1.5 the existing a-posteriori error analysis is extended to our state-constrained control problem. The combination of the a-posteriori error estimation and OS-POD is explained in Section 1.6. In Section 1.7 we propose two algorithms to solve the reduced optimal control problem. Numerical examples are presented in Section 1.8.

\subsection{The state-constrained optimal control problem}

Suppose that $\Omega \subset \mathbb{R}^{d}, d \in\{1,2,3\}$, is an open and bounded domain with Lipschitzcontinuous boundary $\Gamma=\partial \Omega$. Let $V$ be a Hilbert space with $H_{0}^{1}(\Omega) \subset V \subset H^{1}(\Omega)$. We endow the Hilbert spaces $H=L^{2}(\Omega)$ and $V$ with the usual inner products

$$
\langle\varphi, \psi\rangle_{H}=\int_{\Omega} \varphi \psi \mathrm{d} x, \quad\langle\varphi, \psi\rangle_{V}=\int_{\Omega} \varphi \psi+\nabla \varphi \cdot \nabla \psi \mathrm{d} x
$$

Let $T>0$ be the final time. We introduce a continuous bilinear form $a(\cdot, \cdot): V \times V \rightarrow$ $\mathbb{R}$ satisfying

$$
a(\varphi, \varphi) \geq \alpha_{1}\|\varphi\|_{V}^{2}-\alpha_{2}\|\varphi\|_{H}^{2} \quad \text { for all } \varphi \in V
$$

for constants $\alpha_{1}>0$ and $\alpha_{2} \geq 0$. Let us mention that the results can be extended easily to time-dependent bilinear forms in a straightforward way. Recall the Hilbert space $W(0, T)=\left\{\varphi \in L^{2}(0, T ; V) \mid \varphi_{t} \in L^{2}\left(0, T ; V^{\prime}\right)\right\}$ endowed with the common inner product [4, pp. 472-479]. Let $\mathcal{D}$ be a bounded subset of $\mathbb{R}^{\mathrm{d}}$ with $\mathrm{d} \in \mathbb{N}$. Then the control space is given by $\mathcal{U}=L^{2}\left(\mathcal{D} ; \mathbb{R}^{m}\right)$ for $m \in \mathbb{N}$. By $\mathcal{U}_{\text {ad }} \subset \mathcal{U}$ we define 
the closed, convex and bounded subset $\mathcal{U}_{\mathrm{ad}}=\left\{u \in \mathcal{U} \mid u_{a} \leq u \leq u_{b}\right.$ in $\left.\mathcal{U}\right\}$, where $u_{a}, u_{b} \in \mathcal{U}$ holds with $u_{a} \leq u_{b}$. In particular, we identify $\mathcal{U}$ with its dual space $\mathcal{U}^{\prime}$. For $u \in \mathcal{U}_{\mathrm{ad}}, y_{\circ} \in H$ and $f \in L^{2}\left(0, T ; V^{\prime}\right)$ we consider the linear evolution problem

$$
\begin{array}{rlrl}
\frac{\mathrm{d}}{\mathrm{d} t}\langle y(t), \varphi\rangle_{H}+a(y(t), \varphi) & =\langle(f+\mathscr{B} u)(t), \varphi\rangle_{V^{\prime}, V} \forall \varphi \in V \text { in }(0, T], \\
y(0) & =y_{\circ} & \text { in } H,
\end{array}
$$

where $\langle\cdot, \cdot\rangle_{V^{\prime}, V}$ stands for the dual pairing between $V$ and its dual space $V^{\prime}$ and $\mathscr{B}: U \rightarrow L^{2}\left(0, T ; V^{\prime}\right)$ is a continuous, linear operator. It is known that for every $f \in L^{2}\left(0, T ; V^{\prime}\right), u \in \mathcal{U}$ and $y_{\circ} \in H$ there is a unique weak solution $y \in W(0, T)$ satisfying (1.1) and

$$
\|y\|_{W(0, T)} \leq C\left(\left\|y_{\circ}\right\|_{H}+\|f\|_{L^{2}\left(0, T ; V^{\prime}\right)}+\|u\|_{u}\right)
$$

for a constant $C>0$ which is independent of $y_{\circ}, f$ and $u$. For a proof of the existence of a unique solution we refer to [4, pp. 512-520]. The a-priori error estimate follows from standard variational techniques and energy estimates.

Remark 1. Let $\hat{y} \in W(0, T)$ be the unique solution to the problem

$$
\frac{\mathrm{d}}{\mathrm{d} t}\langle y(t), \varphi\rangle_{H}+a(y(t), \varphi)=\langle f(t), \varphi\rangle_{V^{\prime}, V} \forall \varphi \in V \text { in }(0, T], \quad y(0)=y_{\circ} \text { in } H .
$$

We introduce the bounded, linear solution operator $\mathscr{S}: L^{2}\left(0, T ; V^{\prime}\right) \rightarrow W(0, T)$ : for $g \in L^{2}\left(0, T ; V^{\prime}\right)$ the function $\mathscr{S} g \in W(0, T)$ is the unique solution to

$$
\frac{\mathrm{d}}{\mathrm{d} t}\langle y(t), \varphi\rangle_{H}+a(y(t), \varphi)=\langle g(t), \varphi\rangle_{V^{\prime}, V} \forall \varphi \in V \text { in }(0, T], \quad y(0)=0 \text { in } H .
$$

Then, the unique solution to (1.1) is given by $y=\hat{y}+\mathscr{S} \mathscr{B} u$.

We set $\mathcal{W}=L^{2}\left(0, T ; \mathbb{R}^{n}\right)$. Let us introduce the set of admissible states

$$
\tilde{y}_{\text {ad }}=\left\{y \in W(0, T) \mid y_{a} \leq \mathscr{I} y \leq y_{b} \text { in } \mathcal{W}\right\},
$$

where $\mathscr{I}: L^{2}(0, T ; V) \rightarrow \mathcal{W}$ is a bounded, linear operator with $n \in \mathbb{N}, y_{a}, y_{b} \in \mathcal{W}$ with $y_{a} \leq y_{b}$. It follows that $\tilde{y}_{\text {ad }}$ is closed and convex in $W(0, T)$. We introduce the Hilbert space $\tilde{X}=W(0, T) \times \mathcal{U}$ endowed with the natural product topology. Moreover, we define the closed and convex subset $\tilde{X}_{\mathrm{ad}}=\tilde{y}_{\mathrm{ad}} \times \mathcal{U}_{\mathrm{ad}} \subset \tilde{X}$. The cost function $\tilde{J}$ : $\tilde{x} \rightarrow \mathbb{R}$ is given by

$$
\tilde{J}(y, u)=\frac{\sigma_{\Omega}}{2}\left\|y(T)-y_{\Omega}\right\|_{H}^{2}+\frac{\sigma_{Q}}{2} \int_{0}^{T}\left\|y(t)-y_{Q}(t)\right\|_{H}^{2} \mathrm{~d} t+\frac{\sigma_{u}}{2}\|u\|_{\mathcal{U}}^{2}
$$

for $x=(y, u) \in \tilde{X}$, where $\sigma_{Q}, \sigma_{\Omega}$ are nonnegative weighting parameters, $\sigma_{u}>0$ is a regularization parameter and $y_{Q} \in L^{2}(0, T ; H), y_{\Omega} \in H$ are given desired states. Then, we consider the following convex optimal control problem 


$$
\min \tilde{J}(x) \quad \text { subject to (s.t.) } \quad x \in \mathcal{F}(\mathbf{P})
$$

with the set $\mathcal{F}(\mathbf{P})=\left\{(\hat{y}+\mathscr{S} \mathscr{B} u, u) \in \tilde{X}_{\text {ad }}\right\}$ of feasible solutions. By (1.2) the cost functional is radially unbounded. Since $J$ is weakly lower semicontinuous, $(\mathbf{P})$ admits a global optimal solution $\bar{x}=(\bar{y}, \bar{u})$ provided $\mathcal{F}(\mathbf{P})$ is nonempty. Since $\sigma_{u}>0$ holds, $\bar{x}$ is uniquely determined. Uniqueness follows from the strict convexity properties of the objective functional on $\tilde{X}_{\text {ad }}$. For a proof we refer to [12, Section 1.5.2] or [24], for instance.

Example 1 (Boundary control without state constraints). For $T>0$ we set $Q=$ $(0, T) \times \Omega$ and $\Sigma=(0, T) \times \Gamma$. Let $V=H^{1}(\Omega)$. For the control space we choose $\mathcal{D}=\Sigma$ and $m=1$, i.e., $\mathcal{U}=L^{2}(\Sigma)$. Then, for given control $u \in \mathcal{U}$ and initial condition $y_{\circ} \in H$ we consider

$$
\begin{aligned}
c_{p} y_{t}(t, x)-\Delta y(t, x) & =\tilde{f}(t, x) & & \text { in } Q, \\
\frac{\partial y}{\partial n}(t, x)+q y(t, x) & =u(t, x) & & \text { on } \Sigma, \\
y(0, x) & =y_{\circ}(x) & & \text { in } \Omega .
\end{aligned}
$$

In (1.4) we suppose $c_{p}>0, q \geq 0$ and $\tilde{f} \in L^{2}(0, T ; H)$. Setting $f=\tilde{f} / c_{p}$, introducing the bounded (symmetric) bilinear form $a: V \times V \rightarrow \mathbb{R}$ by

$$
a(\varphi, \psi)=\frac{1}{c_{p}} \int_{\Omega} \nabla \varphi(x) \cdot \nabla \psi(x) \mathrm{d} x+\frac{q}{c_{p}} \int_{\Gamma} \varphi(x) \psi(x) \mathrm{d} x \quad \text { for } \varphi, \psi \in V
$$

and the linear, bounded operator $\mathscr{B}: U \rightarrow L^{2}\left(0, T ; V^{\prime}\right)$ by

$$
\langle(\mathscr{B} u)(t), \varphi\rangle_{V^{\prime}, V}=\frac{1}{c_{p}} \int_{\Gamma} u(t, x) \varphi(x) \mathrm{d} x \quad \text { for } \phi \in V, t \in[0, T]
$$

then the weak formulation of (1.4) can be expressed in the form (1.1). More details on this example one can found in [6].

Example 2 (Distributed control with state constraints). Let $\Omega, \Gamma, T, Q, \Sigma$ as in Example 1 . Let $\chi_{i} \in H, 1 \leq i \leq m$, denote given control shape functions. For the control space we choose $\mathcal{D}=(0, T)$ and set $\mathcal{U}=L^{2}\left(0, T ; \mathbb{R}^{m}\right)$. Then, for given control $u \in \mathcal{U}$, initial condition $y_{\circ} \in H$ and inhomogeneity $f \in L^{2}(0, T ; H)$ we consider the linear heat equation

$$
\begin{aligned}
y_{t}(t, x)-v \Delta y(t, x)+\beta \cdot \nabla y(t, x) & =f(t, x)+\sum_{i=1}^{m} u_{i}(t) \chi_{i}(x), & & \text { in } Q, \\
y(t, x) & =0 & & \text { on } \Sigma, \\
y(0, x) & =y_{\circ}(x) & & \text { in } \Omega .
\end{aligned}
$$

with $v>0$ and $\beta \in \mathbb{R}^{d}$. We introduce the bounded (symmetric) bilinear form 


$$
a(\varphi, \psi)=\int_{\Omega} \nabla \varphi \cdot \nabla \psi \mathrm{d} x \quad \text { for } \varphi, \psi \in V
$$

and the bounded, linear operator $\mathscr{B}: \mathcal{U} \rightarrow L^{2}(0, T ; H) \hookrightarrow L^{2}\left(0, T ; V^{\prime}\right)$ as

$$
(\mathscr{B} u)(t, x)=\sum_{i=1}^{m} u_{i}(t) \chi_{i}(x) \quad \text { for }(t, x) \in Q \text { and } u \in \mathcal{U} \text {. }
$$

It follows that the weak formulation of (1.5) can be expressed in the form (1.1). We choose certain shape functions $\pi_{1}, \ldots, \pi_{n} \in H$ and introduce the operator $\mathscr{I}$ : $L^{2}(0, T ; V) \rightarrow \mathcal{W}$ by

$$
(\mathscr{I} \varphi)(t)=\left(\begin{array}{c}
\left(\mathscr{I}_{1} \varphi\right)(t) \\
\vdots \\
\left(\mathscr{I}_{n} \varphi\right)(t)
\end{array}\right) \quad \text { with } \quad\left(\mathscr{I}_{i} \varphi\right)(t)=\int_{\Omega} \pi_{i}(x) \varphi(t, x) \mathrm{d} x
$$

for $\varphi \in L^{2}(0, T ; V)$. Then, the state constraints have the form

$$
y_{a i}(t) \leq \int_{\Omega} \pi_{i}(x) y(t, x) \mathrm{d} x \leq y_{b i}(t) \quad \text { in }[0, T] \text { and for } 1 \leq i \leq n,
$$

where $(y, w) \in W(0, T) \times \mathcal{W}$ holds; see also [7].

\subsection{The Lavrentiev regularization}

It is well-known that the (sufficient) first-order optimality conditions for (P) involve a measure-valued Lagrange multiplier associated with the state constraint $\bar{y} \in \tilde{y}_{\text {ad }}$; see [12, Section 1.7.3]. To develop a fast numerical solution methods (by combining semismooth Newton techniques with reduced-order modelling) we apply a Lavrentiev regularization of the state constraints. For that purpose we introduce an additional (artificial) control variable and approximate the pure state by mixed controlstate constraints, which enjoy $L^{2}$-regularity; see [23].

Instead of $\tilde{X}$ we consider the Hilbert space $\mathcal{X}=W(0, T) \times \mathcal{U} \times \mathcal{W}$, again supplied with the product topology. For given $\varepsilon>0$ the subset $\tilde{X}_{\text {ad }}$ is replaced by the closed and convex subset

$$
X_{\mathrm{ad}}^{\mathcal{E}}=\left\{(y, u, w) \in X \mid y_{a} \leq \varepsilon w+\mathscr{I} y \leq y_{b} \text { in } \mathcal{W}, u \in \mathcal{U}_{\mathrm{ad}}\right\} .
$$

For a chosen weight $\sigma_{w}>0$ we also extend the cost functional $\tilde{J}$ by defining $J: X \rightarrow$ $\mathbb{R}$ with

$$
J(y, u, w)=\tilde{J}(y, u)+\frac{\sigma_{w}}{2}\|w\|_{\mathcal{W}}^{2}, \quad x=(y, u, w) \in X .
$$

Now the regularized optimal control problem has the following form

$$
\min J(x) \quad \text { s.t. } \quad x \in \mathcal{F}\left(\mathbf{P}^{\varepsilon}\right)
$$


with the feasible set $\mathcal{F}\left(\mathbf{P}^{\varepsilon}\right)=\left\{(\hat{y}+\mathscr{S} \mathscr{B} u, u, w) \in X_{\text {ad }}^{\varepsilon}\right\}$. If $\mathcal{F}\left(\mathbf{P}^{\varepsilon}\right) \neq \emptyset$ holds, it follows by similar arguments as above that $\left(\mathbf{P}^{\varepsilon}\right)$ possesses a unique global optimal solution $\bar{x}$.

Let us define the control space $\mathcal{V}=\mathcal{U} \times \mathcal{W}$. We introduce the reduced cost functional $\hat{J}$ by $\hat{J}(v)=J(\hat{y}+\mathscr{S} \mathscr{B} u, u, w)$ for $v=(u, w) \in \mathcal{V}$. By Remark 1 the solution to (1.1) can be expressed as $y=\hat{y}+\mathscr{S} \mathscr{B} u$. Thus, the set of admissible controls is given by

$$
\mathcal{V}_{\mathrm{ad}}^{\varepsilon}=\left\{v=(u, w) \in \mathcal{V} \mid u \in \mathcal{U}_{\mathrm{ad}} \text { and } \hat{y}_{a} \leq \varepsilon w+\mathscr{I} \mathscr{S} \mathscr{B} u \leq \hat{y}_{b} \text { in } \mathcal{W}\right\}
$$

with $\hat{y}_{a}=y_{a}-\mathscr{I} \hat{y}$ and $\hat{y}_{b}=y_{b}-\mathscr{I} \hat{y}$. Now, $\left(\mathbf{P}^{\varepsilon}\right)$ is equivalent to the reduced problem

$$
\min \hat{J}(v) \quad \text { s.t. } \quad v \in \mathcal{V}_{\text {ad }}^{\varepsilon} .
$$

The control $\bar{v}=(\bar{u}, \bar{w})$ is the unique solution to $\left(\hat{\mathbf{P}}^{\varepsilon}\right)$ if and only if $\bar{x}=(\hat{y}+\mathscr{S} \mathscr{B} \bar{u}, \bar{v})$ is the unique solution to $\left(\mathbf{P}^{\varepsilon}\right)$.

Next we formulate first-order sufficient optimality conditions for $\left(\mathbf{P}^{\varepsilon}\right)$ (see [24], for instance):

Theorem 1. Suppose that the feasible set $\mathcal{F}\left(\mathbf{P}^{\varepsilon}\right)$ is nonempty. The point $\bar{x}=(\bar{y}, \bar{u}, \bar{w}) \in$ $X_{\mathrm{ad}}^{\varepsilon}$ is a (global) optimal solution to $\left(\mathbf{P}^{\varepsilon}\right)$ if and only if there are unique Lagrange multipliers $\left(\bar{p}, \bar{\lambda}_{u}, \bar{\lambda}_{y}\right) \in X$ satisfying the dual equations

$$
\begin{gathered}
-\frac{\mathrm{d}}{\mathrm{d} t}\langle\bar{p}(t), \varphi\rangle_{H}+a(\varphi, \bar{p}(t))+\left\langle\left(\mathscr{I}^{\star} \bar{\lambda}_{y}\right)(t), \varphi\right\rangle_{V^{\prime}, V}=\sigma_{Q}\left\langle\left(y_{Q}-\bar{y}\right)(t), \varphi\right\rangle_{H} \\
\forall \varphi \in V \text { in }[0, T), \quad \bar{p}(T)=\sigma_{\Omega}\left(y_{\Omega}-\bar{y}(T)\right) \text { in } H,
\end{gathered}
$$

and the optimality conditions

$$
\sigma_{u} \bar{u}-\mathscr{B}^{\star} \bar{p}+\bar{\lambda}_{u}=0 \text { in } \mathcal{U}, \quad \sigma_{w} \bar{w}+\varepsilon \bar{\lambda}_{y}=0 \text { in } \mathcal{W},
$$

where $\mathscr{I}^{\star}: \mathcal{W} \rightarrow L^{2}\left(0, T ; V^{\prime}\right)$ and $\mathscr{B}^{\star}: L^{2}(0, T ; V) \rightarrow \mathcal{U}$ denote the adjoint operators of $\mathscr{I}$ and $\mathscr{B}$, respectively. For the Lagrange multipliers $\bar{\lambda}_{u}$ and $\bar{\lambda}_{y}$ we have

$$
\begin{array}{ll}
\bar{\lambda}_{u}=\max \left(0, \bar{\lambda}_{u}+\gamma_{u}\left(\bar{u}-u_{b}\right)\right)+\min \left(0, \bar{\lambda}_{u}+\gamma_{u}\left(\bar{u}-u_{a}\right)\right) & \text { in } \mathcal{U}, \\
\bar{\lambda}_{y}=\max \left(0, \bar{\lambda}_{y}+\gamma_{w}\left(\varepsilon \bar{w}+\mathscr{I} \bar{y}-y_{b}\right)\right)+\min \left(0, \bar{\lambda}_{y}+\gamma_{w}\left(\varepsilon \bar{w}+\mathscr{I} \bar{y}-y_{a}\right)\right) & \text { in } \mathcal{W},
\end{array}
$$

where $\gamma_{u}, \gamma_{w}>0$ are arbitrarily chosen.

Remark 2. 1) Analogous to Remark 1 we split the adjoint variable into one part depending on the fixed desired states and into two other parts, which depend linearly on the control variable and on the multiplier $\lambda$. Recall that we have defined $\hat{y}$ as well as the operator $\mathscr{S}$ in Remark 1 . For given $y_{Q} \in L^{2}(0, T ; H)$ and $y_{\Omega} \in H$ let $\hat{p} \in W(0, T)$ denote the unique solution to the adjoint equation 


$$
\begin{aligned}
-\frac{\mathrm{d}}{\mathrm{d} t}\langle\hat{p}(t), \varphi\rangle_{H}+a(\varphi, \hat{p}(t)) & =\sigma_{Q}\left\langle\left(y_{Q}-\hat{y}\right)(t), \varphi\right\rangle_{H} & & \forall \varphi \in V \text { in }[0, T), \\
\hat{p}(T) & =\sigma_{\Omega}\left(y_{\Omega}-\hat{y}(T)\right) & & \text { in } H .
\end{aligned}
$$

Further, we define the linear, bounded operators $\mathscr{A}_{1}: \mathcal{U} \rightarrow W(0, T)$ and $\mathscr{A}_{2}: \mathcal{W} \rightarrow$ $W(0, T)$ as follows: for any $u \in \mathcal{U}$ the function $p=\mathscr{A}_{1} u$ is the unique solution to

$$
\begin{aligned}
-\frac{\mathrm{d}}{\mathrm{d} t}\langle p(t), \varphi\rangle_{H}+a(\varphi, p(t)) & =-\sigma_{Q}\langle(\mathscr{S} \mathscr{B} u)(t), \varphi\rangle_{H} & & \forall \varphi \in V \text { in }[0, T), \\
p(T) & =-\sigma_{\Omega}(\mathscr{S} \mathscr{B} u)(T) & & \text { in } H
\end{aligned}
$$

and for given $\lambda \in \mathcal{W}$ the function $p=\mathscr{A}_{2} \lambda$ uniquely solves $p(T)=0$ in $H$ and

$$
-\frac{\mathrm{d}}{\mathrm{d} t}\langle p(t), \varphi\rangle_{H}+a(\varphi, p(t))+\left\langle\left(\mathscr{I}^{\star} \lambda_{y}\right)(t), \varphi\right\rangle_{V^{\prime}, V}=0 \quad \forall \varphi \in V \text { in }[0, T) .
$$

Then, the solution to (1.7) can be expressed as $\bar{p}=\hat{p}+\mathscr{A}_{1} \bar{u}+\mathscr{A}_{2} \bar{\lambda}_{y}$.

2) To solve $\left(\mathbf{P}^{\varepsilon}\right)$ numerically for fixed $\varepsilon>0$ we use a primal-dual active set strategy. This method is equivalent to a locally superlinearly convergent semi-smooth Newton algorithm applied to the first-order optimality conditions $[8,9,10]$.

\subsection{The POD method}

Let $z$ be either the space $H$ or the space $V$. In $z$ we denote by $\langle\cdot, \cdot\rangle_{z}$ and $\|\cdot\|_{z}=$ $\langle\cdot, \cdot\rangle_{Z}^{1 / 2}$ the inner product and the associated norm, respectively. For fixed $\wp \in \mathbb{N}$ let the so-called snapshots $z^{k}(t) \in Z$ be given for $t \in[0, T]$ and $1 \leq k \leq \wp$. To avoid a trivial case we suppose that at least one of the $z^{k}$ 's is nonzero. Then, we introduce the linear subspace

$$
z^{\wp}=\operatorname{span}\left\{z^{k}(t) \mid t \in[0, T] \text { and } 1 \leq k \leq \wp\right\} \subset z
$$

with dimension $\mathfrak{d} \geq 1$. We call the set $Z^{\text {s }}$ snapshot subspace. The method of POD consists in choosing a complete orthonormal basis $\left\{\psi_{i}\right\}_{i=1}^{\infty}$ in $z$ such that for every $\ell \leq \mathfrak{d}$ the mean square error between the $\wp$ elements $z^{k}$ and their corresponding $\ell$-th partial Fourier sum is minimized:

$$
\left\{\begin{array}{l}
\min \sum_{k=1}^{\wp} \int_{0}^{T}\left\|z^{k}(t)-\sum_{i=1}^{\ell}\left\langle z^{k}(t), \psi_{i}\right\rangle_{z} \psi_{i}\right\|_{z}^{2} \mathrm{~d} t \\
\text { s.t. }\left\{\psi_{i}\right\}_{i=1}^{\ell} \subset Z \text { and }\left\langle\psi_{i}, \psi_{j}\right\rangle_{z}=\delta_{i j}, 1 \leq i, j \leq \ell .
\end{array}\right.
$$

In (1.9) the symbol $\delta_{i j}$ denotes the Kronecker symbol satisfying $\delta_{i i}=1$ and $\delta_{i j}=0$ for $i \neq j$. An optimal solution $\left\{\bar{\psi}_{i}^{n}\right\}_{i=1}^{\ell}$ to (1.9) is called a POD basis of rank $\ell$. 
Remark 3. In real computations, we do not have the whole trajectories $z^{k}(t)$ at hand for all $t \in[0, T]$ and $1 \leq k \leq \wp$. Here we apply a discrete variant of the POD method; see $[7,16]$ for more details.

To solve (1.9) we define the linear operator $\mathscr{R}: \mathcal{Z} \rightarrow Z^{\wp}$ as follows:

$$
\mathscr{R} \psi=\sum_{k=1}^{\wp} \int_{0}^{T}\left\langle\psi, z^{k}(t)\right\rangle_{z} z^{k}(t) \mathrm{d} t \quad \text { for } \psi \in Z
$$

Then, $\mathscr{R}$ is a compact, nonnegative and selfadjoint operator. Suppose that $\left\{\bar{\lambda}_{i}\right\}_{i=1}^{\infty}$ and $\left\{\bar{\psi}_{i}\right\}_{i=1}^{\infty}$ denote the nonnegative eigenvalues and associated orthonormal eigenfunctions of $\mathscr{R}$ satisfying

$$
\mathscr{R} \bar{\psi}_{i}=\bar{\lambda}_{i} \bar{\psi}_{i}, \quad \bar{\lambda}_{1} \geq \ldots \geq \bar{\lambda}_{\mathfrak{d}}>\bar{\lambda}_{\mathfrak{d}+1}=\ldots=0 .
$$

Then, for every $\ell \leq \mathfrak{d}$ the first $\ell$ eigenfunctions $\left\{\bar{\psi}_{i}\right\}_{i=1}^{\ell}$ solve (1.9) and

$$
\sum_{k=1}^{\wp} \int_{0}^{T}\left\|z^{k}(t)-\sum_{i=1}^{\ell}\left\langle z^{k}(t), \bar{\psi}_{i}\right\rangle_{z} \bar{\psi}_{i}\right\|_{z}^{2} \mathrm{~d} t=\sum_{i=\ell+1}^{\mathfrak{d}} \bar{\lambda}_{i} .
$$

For more details we refer the reader to $[11,13]$ and $[7$, Chapter 2], for instance.

Remark 4. a) In the context of the optimal control problem $\left(\mathbf{P}^{\varepsilon}\right)$ a reasonable choice for the snapshots is $z^{1}=y$ and $z^{2}=p$. Utilizing new POD error estimates for evolution problems $[3,20]$ and optimal control problems [14, 25] convergence and rate of convergence results are derived for linear-quadratic control constrained problems in [7] for the choices $z=H$ and $z=V$.

b) For the numerical realization the space $z$ has to be discretized by, e.g., finite element discretizations. In this case the Hilbert space $Z$ has to be replaced by an Euclidean space $\mathbb{R}^{l}$ endowed with a weighted inner product; see [7].

If a POD basis $\left\{\psi_{i}\right\}_{i=1}^{\ell}$ of rank $\ell$ is computed, we set $V^{\ell}=\operatorname{span}\left\{\psi_{1}, \ldots, \psi_{\ell}\right\}$. Then, one can derive a reduced-order model (ROM) for (1.1): for any $g \in L^{2}\left(0, T ; V^{\prime}\right)$ the function $q^{\ell}=\mathscr{S}^{\ell} g$ is given by $q^{\ell}(0)=0$ in $H$ and

$$
\frac{\mathrm{d}}{\mathrm{d} t}\left\langle q^{\ell}(t), \psi\right\rangle_{H}+a\left(q^{\ell}(t), \psi\right)=\langle g(t), \psi\rangle_{V^{\prime}, V} \quad \forall \psi \in V^{\ell} \text { in }(0, T] .
$$

For any $u \in \mathcal{U}_{\text {ad }}$ the POD approximation $y^{\ell}$ for the state solution is $y^{\ell}=\hat{y}+\mathscr{S}^{\ell} \mathscr{B} u$. Analogously, a ROM can be derived for the adjoint equation; see, e.g., [7]. The POD Galerkin approximation of $\left(\hat{\mathbf{P}}^{\varepsilon}\right)$ is given by

$$
\min J^{\ell}(v)=J\left(\hat{y}+\mathscr{S}^{\ell} \mathscr{B} u, v\right) \quad \text { s.t. } \quad v=(u, w) \in \mathcal{V}_{\mathrm{ad}}^{\varepsilon, \ell} \quad\left(\hat{\mathbf{P}}^{\varepsilon, \ell}\right)
$$

where the set of admissible controls is

$$
\mathcal{V}_{\mathrm{ad}}^{\varepsilon, \ell}=\left\{v=(u, w) \in \mathcal{V} \mid u \in \mathcal{U}_{\mathrm{ad}} \text { and } \hat{y}_{a} \leq \varepsilon w+\mathscr{I} \mathscr{S}^{\ell} \mathscr{B} u \leq \hat{y}_{b} \text { in } \mathcal{W}\right\} .
$$




\subsection{A-posteriori error analysis}

Let us consider (P) with control, but no state constraints. Based on a perturbation argument [5] it is derived in [25] how far the suboptimal POD control $\bar{u}^{\ell}$, computed on the basis of the POD model, is from the (unknown) exact $\bar{u}$. Then, the error estimate reads as follows:

$$
\left\|\bar{u}^{\ell}-\bar{u}\right\|_{u} \leq \frac{1}{\sigma_{u}}\left\|\zeta^{\ell}\right\|_{u},
$$

where the computable perturbation function $\zeta^{\ell} \in \mathcal{U}$ is given by

$$
\zeta^{\ell}= \begin{cases}-\min \left(0, \sigma_{u} \bar{u}^{\ell}-\mathscr{B}^{\star} \tilde{p}^{\ell}\right) & \text { in } \mathcal{A}_{a}^{\ell}=\left\{s \in \mathcal{D} \mid \bar{u}^{\ell}(s)=u_{a}(s)\right\}, \\ -\max \left(0, \sigma_{u} \bar{u}^{\ell}-\mathscr{B}^{\star} \tilde{p}^{\ell}\right) & \text { in } \mathcal{A}_{b}^{\ell}=\left\{s \in \mathcal{D} \mid \bar{u}^{\ell}(s)=u_{b}(s)\right\} \\ -\left(\sigma_{u} \bar{u}^{\ell}-\mathscr{B}^{\star} \tilde{p}^{\ell}\right) & \text { in } \mathcal{D} \backslash\left(\mathcal{A}_{a}^{\ell} \cup \mathcal{A}_{b}^{\ell}\right),\end{cases}
$$

with $\tilde{p}^{\ell}=\hat{p}+\mathscr{A}_{1} \bar{u}^{\ell}$. It is shown in $[7,25]$ that $\left\|\zeta^{\ell}\right\|_{u}$ tends to zero as $\ell$ tends to infinity. Hence, increasing the number of POD ansatz functions leads to more accurate POD suboptimal controls.

Estimate (1.12) can be generalized for the mixed control-state constraints. Firstorder sufficient optimality conditions for $\left(\hat{\mathbf{P}}^{\varepsilon}\right)$ are of the form

$$
\left\langle\hat{J}^{\prime}(\bar{v}), v-\bar{v}\right\rangle_{\mathcal{V}} \geq 0 \quad \text { for all } v \in \mathcal{V}_{\mathrm{ad}}^{\varepsilon}
$$

where the gradient at a point $v=(u, w) \in \mathcal{V}$ is given by [24]

$$
\left\langle\hat{J}^{\prime}(v), v_{\delta}\right\rangle_{\mathcal{V}}=\left\langle\sigma_{u} u-\mathscr{B}^{\star}\left(\hat{p}+\mathscr{A}_{1} u\right), u_{\delta}\right\rangle_{\mathcal{U}}+\left\langle\sigma_{w} w_{\delta}, w_{\delta}\right\rangle_{\mathcal{W}} \forall v_{\delta}=\left(u_{\delta}, w_{\delta}\right) \in \mathcal{V}
$$

Let us introduce the bounded, linear transformation $\mathscr{T}: \mathcal{V} \rightarrow \mathcal{V}$ as

$$
\mathscr{T}(v)=(u, \varepsilon w+\mathscr{I} \mathscr{S} \mathscr{B} u) \quad \text { for } v=(u, w) \in \mathcal{V}
$$

We assume that $\mathscr{T}$ is continuously invertible. For sufficient conditions we refer to [8, Lemma 2.1]. Then, $v=(u, w)$ belongs to $\mathcal{V}_{\text {ad }}^{\varepsilon}$ if and only if $\mathfrak{v}=(\mathfrak{u}, \mathfrak{w})=\mathscr{T}(v)$ satisfies

$$
u_{a} \leq \mathfrak{u} \leq u_{b} \text { in } \mathcal{U} \quad \text { and } \quad \hat{y}_{a} \leq \mathfrak{w} \leq \hat{y}_{b} \text { in } \mathcal{W} .
$$

Notice that (1.13) can be expressed equivalently as

$$
\left\langle\mathscr{T}^{-\star} \hat{J}^{\prime}\left(\mathscr{T}^{-1} \overline{\mathfrak{v}}\right), \mathfrak{v}-\overline{\mathfrak{v}}\right\rangle_{\mathcal{V}} \geq 0 \quad \text { for all } \mathfrak{v} \in \mathcal{V} \text { satisfying (1.15), }
$$

where $\mathscr{T}^{-\star}$ denotes the inverse of the operator $\mathscr{T}^{\star}$. Suppose that $\bar{v}^{\ell}=\left(\bar{u}^{\ell}, \bar{w}^{\ell}\right) \in \mathcal{V}_{\text {ad }}^{\varepsilon, \ell}$ is the solution to $\left(\hat{\mathbf{P}}^{\varepsilon, \ell}\right)$. Our goal is to estimate the norm

$$
\left\|\bar{v}-\bar{v}^{\ell}\right\|_{\mathcal{V}}
$$


without the knowledge of the optimal solution $\bar{v}=\mathscr{T}^{-1} \overline{\mathfrak{v}}$. We set $\overline{\mathfrak{v}}^{\ell}=\mathscr{T} \bar{v}^{\ell}=$ $\left(\bar{u}^{\ell}, \varepsilon \bar{w}^{\ell}+\mathscr{I} \mathscr{S} \mathscr{B} \bar{u}^{\ell}\right)$. If $\bar{v}^{\ell} \neq \bar{v}$ holds, then $\overline{\mathfrak{v}}^{\ell} \neq \overline{\mathfrak{v}}$. In particular, $\bar{v}^{\ell}$ does not satisfy the sufficient optimality condition (1.13). However, there exists a function $\zeta^{\ell} \in \mathcal{V}$ such that

$$
\left\langle\mathscr{T}^{-\star} \hat{J}^{\prime}\left(\mathscr{T}^{-1} \overline{\mathfrak{v}}^{\ell}\right)+\zeta^{\ell}, \mathfrak{v}-\overline{\mathfrak{v}}^{\ell}\right\rangle_{\mathcal{V}} \geq 0 \quad \text { for all } \mathfrak{v} \in \mathcal{V} \text { satisfying (1.15). }
$$

Choosing $\mathfrak{v}=\overline{\mathfrak{v}}^{\ell}$ in (1.16), $\mathfrak{v}=\overline{\mathfrak{v}}$ in (1.17) and adding both inequality we infer that

$$
\begin{aligned}
0 & \leq\left\langle\mathscr{T}^{-\star}\left(\hat{J}^{\prime}\left(\mathscr{T}^{-1} \overline{\mathfrak{v}}^{\ell}\right)+\mathscr{T}^{\star} \zeta^{\ell}-\hat{J}^{\prime}\left(\mathscr{T}^{-1} \overline{\mathfrak{v}}\right)\right), \overline{\mathfrak{v}}-\overline{\mathfrak{v}}^{\ell}\right\rangle_{\mathcal{V}} \\
& =\left\langle\hat{J}^{\prime}\left(\bar{v}^{\ell}\right)-\bar{J}^{\prime}(\bar{v})+\mathscr{T}^{\star} \zeta^{\ell}, \mathscr{T}^{-1}\left(\overline{\mathfrak{v}}-\overline{\mathfrak{v}}^{\ell}\right)\right\rangle_{\mathcal{V}} \\
& =\left\langle\left(\sigma_{u}\left(\bar{u}^{\ell}-\bar{u}\right)-\mathscr{B}^{\star} \mathscr{A}_{1}\left(\bar{u}^{\ell}-\bar{u}\right), \sigma_{w}\left(\bar{w}^{\ell}-\bar{w}\right)\right)+\mathscr{T}^{\star} \zeta^{\ell}, \bar{v}-\bar{v}^{\ell}\right\rangle_{\mathcal{V}} \\
& \leq-\sigma\left\|\bar{v}-\bar{v}^{\ell}\right\|_{\mathcal{V}}^{2}+\left\langle\mathscr{B}^{\star} \mathscr{A}_{1}\left(\bar{u}-\bar{u}^{\ell}\right), \bar{u}-\bar{u}^{\ell}\right\rangle_{\mathcal{U}}+\left\langle\mathscr{T}^{\star} \zeta^{\ell}, \bar{v}-\bar{v}^{\ell}\right\rangle_{\mathcal{V}}
\end{aligned}
$$

with $\sigma=\min \left(\sigma_{u}, \sigma_{w}\right)>0$. In [8, Lemma 2.2] it is shown that $\left\langle\mathscr{B}^{\star} \mathscr{A}_{1}\left(\bar{u}-\bar{u}^{\ell}\right), \bar{u}-\right.$ $\left.\bar{u}^{\ell}\right\rangle_{u} \leq 0$ holds. Consequently,

$$
0 \leq-\sigma\left\|\bar{v}-\bar{v}^{\ell}\right\|_{\mathcal{V}}^{2}+\left\langle\mathscr{T}^{\star} \zeta^{\ell}, \bar{v}-\bar{v}^{\ell}\right\rangle_{\mathcal{V}} \leq-\sigma\left\|\bar{v}-\bar{v}^{\ell}\right\|_{\mathcal{V}}^{2}+\left\|\mathscr{T}^{\star} \zeta\right\|_{\mathcal{V}}\left\|\bar{v}-\bar{v}^{\ell}\right\|_{\mathcal{V}}
$$

which implies the following proposition.

Proposition 1. Let the operator $\mathscr{T}$ - introduced in (1.14) - possess a bounded inverse. Suppose that $\bar{v}$ and $\bar{v}^{\ell}$ are the optimal solution to $\left(\hat{\mathbf{P}}^{\varepsilon}\right)$ and $\left(\hat{\mathbf{P}}^{\varepsilon, \ell}\right)$, respectively, satisfying $\overline{\mathfrak{v}}^{\ell}=\mathscr{T} \bar{v}^{\ell} \in \mathcal{V}_{\mathrm{ad}}^{\varepsilon}$. Then, there is a perturbation $\zeta^{\ell}=\left(\zeta_{u}^{\ell}, \zeta_{w}^{\ell}\right) \in \mathcal{V}$ satisfying

$$
\left\|\bar{v}-\bar{v}^{\ell}\right\|_{\mathcal{V}} \leq \frac{1}{\sigma}\left\|\mathscr{T}^{\star} \zeta^{\ell}\right\|_{\mathcal{V}} \quad \text { with } \sigma=\min \left(\sigma_{u}, \sigma_{w}\right)>0 .
$$

The perturbation $\zeta^{\ell}=\left(\zeta_{u}^{\ell}, \zeta_{w}^{\ell}\right)$ can be computed as follows: Let $\xi^{\ell}=\left(\xi_{u}^{\ell}, \xi_{w}^{\ell}\right)=$ $\mathscr{T}^{-\star} \hat{J}^{\prime}\left(\bar{v}^{\ell}\right) \in \mathcal{V}$. Then, $\xi^{\ell}$ solves the linear system

$$
\left(\begin{array}{cc}
\operatorname{id}_{\mathcal{u}} & 0 \\
\mathscr{B}^{\star} \mathscr{S}^{\star} \mathscr{I}^{\star} & \operatorname{cid}_{\mathcal{W}}
\end{array}\right)\left(\begin{array}{l}
\xi_{u}^{\ell} \\
\xi_{w}^{\ell}
\end{array}\right)=\left(\begin{array}{c}
\sigma_{u} \bar{u}^{\ell}-\mathscr{B}^{\star}\left(\hat{p}+\mathscr{A}_{1} \bar{u}^{\ell}\right) \\
\sigma_{w} \bar{w}^{\ell}
\end{array}\right),
$$

where, e.g., $\mathrm{id}_{\mathcal{U}}: \mathcal{U} \rightarrow \mathcal{U}$ stands for the identity operator. Note that (1.17) can be written as $\left\langle\xi+\zeta, \mathfrak{v}-\overline{\mathfrak{v}}^{\ell}\right\rangle_{\mathcal{V}} \geq 0$ for all $\mathfrak{v} \in \mathcal{V}$ satisfying (1.15). We find

$$
\zeta_{u}^{\ell}= \begin{cases}-\min \left(0, \xi_{u}^{\ell}\right) & \text { in } \mathcal{A}_{a}^{u}=\left\{\bar{u}^{\ell}=u_{a}\right\} \subset \mathcal{U}, \\ -\max \left(0, \xi_{u}^{\ell}\right) & \text { in } \mathcal{A}_{b}^{u}=\left\{\bar{u}^{\ell}=u_{b}\right\} \subset \mathcal{U}, \\ -\xi_{u}^{\ell} & \text { in } \mathcal{U} \backslash\left(\mathcal{A}_{a}^{u} \cup \mathcal{A}_{b}^{u}\right)\end{cases}
$$

and

$$
\zeta_{w}^{\ell}= \begin{cases}-\min \left(0, \xi_{w}^{\ell}\right) & \text { in } \mathcal{A}_{a}^{w}=\left\{\varepsilon \bar{w}^{\ell}+\mathscr{I} \mathscr{S} \mathscr{B} \bar{u}^{\ell}=\hat{y}_{a}\right\} \subset \mathcal{W} \\ -\max \left(0, \xi_{w}^{\ell}\right) & \text { in } \mathcal{A}_{a}^{w}=\left\{\varepsilon \bar{w}^{\ell}+\mathscr{I} \mathscr{S} \mathscr{B} \bar{u}^{\ell}=\hat{y}_{b}\right\} \subset \mathcal{W} \\ -\xi_{w}^{\ell} & \text { in } \mathcal{W} \backslash\left(\mathcal{A}_{a}^{w} \cup \mathcal{A}_{b}^{w}\right)\end{cases}
$$




\subsection{Optimality-system POD}

The accuracy of the reduced-order model can be controlled by the a-posteriori error analysis presented in Section 1.5. However, if the POD basis is created from a reference trajectory containing features which are quite different from those of the optimally controlled trajectory, a rather huge number of POD ansatz functions has to be included in the reduced-order model. This fact may lead to non-efficient reducedorder models and numerical instabilities. To avoid these problems the POD basis is generated in an initialization step utilizing optimality system POD (OS-POD) introduced in [17]. In OS-POD the POD basis is updated in the direction of the minimum of the cost. Recall that the POD basis is computed from the state $y=\hat{y}+\mathscr{S} \mathscr{B} u$ with some control $u^{0} \in \mathcal{U}_{\mathrm{ad}}$. Thus, the reduced-order Galerkin projection depends on the state variable and hence on the control $u$ at which the eigenvalue $\mathscr{R} \psi_{i}=\lambda_{i} \psi_{i}$ for $i=1, \ldots, \ell$ is solved for the basis $\left\{\psi_{i}\right\}_{i=1}^{\ell}$. If the optimal control $\bar{u}$ differs significantly from the initially chosen control $u^{0}$, the POD basis does not reflect the dynamics of the system in a sufficiently accurate manner. Therefore, we consider the extended problem:

$$
\min \hat{J}^{\ell}(v) \text { s.t. }\left\{\begin{array}{l}
v=(u, w) \in \mathcal{V}_{\mathrm{ad}}^{\varepsilon, \ell}, \\
(\psi, \lambda) \text { satisfies }(1.11) \text { for } \wp=1 \text { and } z^{1}=\hat{y}+\mathscr{S} \mathscr{B} u .
\end{array} \quad\left(\hat{\mathbf{P}}_{\mathrm{os}}^{\varepsilon, \ell}\right)\right.
$$

Notice that the first line of the constraints in $\left(\hat{\mathbf{P}}_{\mathrm{OS}}^{\varepsilon, \ell}\right)$ coincides with the constraints in $\left(\hat{\mathbf{P}}^{\varepsilon, \ell}\right)$, whereas the second line of the constraints in $\left(\hat{\mathbf{P}}_{\mathrm{OS}}^{\varepsilon, \ell}\right)$ are the infinitedimensional eigenvalue problem defining the POD basis. For the optimal solution the problem formulation $\left(\hat{\mathbf{P}}_{\mathrm{os}}^{\varepsilon, \ell}\right)$ has the property that the associated POD reduced system is computed from the trajectory corresponding to the optimal control and thus, differently from $\left(\hat{\mathbf{P}}^{\varepsilon, \ell}\right)$, the problem of unmodelled dynamics is removed. Of course, $\left(\hat{\mathbf{P}}_{\mathrm{OS}}^{\varepsilon, \ell}\right)$ is more complicated than $\left(\hat{\mathbf{P}}^{\varepsilon, \ell}\right)$. For practical realization an operator splitting approach is used in [17], where also sufficient conditions are given so that $\left(\hat{\mathbf{P}}_{\text {os }}^{\varepsilon, \ell}\right)$ possesses a unique optimal solution, which can be characterized by first-order necessary optimality conditions; compare [17] for more details. Convergence results for OS-POD are studied in [18]. The combination of OS-POD and a-posteriori error analysis is suggested in [26] and tested successfully in [6]. The resulting strategy is presented in the next section.

\subsection{Algorithms}

For pure control constraints, i.e., $\hat{J}^{\ell}$ depends only on $u$, a variable splitting is proposed, where a good POD basis is initialized by applying a few projected gradient steps [15]. Then, the POD basis is kept fixed and $\left(\hat{\mathbf{P}}^{\varepsilon, \ell}\right)$ is solved. If the a-posteriori error estimator $\left\|\zeta^{\ell}\right\|_{u} / \sigma_{u}$ is too large (compare (1.12)), the number $\ell$ of POD basis elements is increased and a new solution to $\left(\hat{\mathbf{P}}^{\varepsilon, \ell}\right)$ is computed. This process 
is repeated until we obtain convergence; see Algorithm 1. Let us mention that we

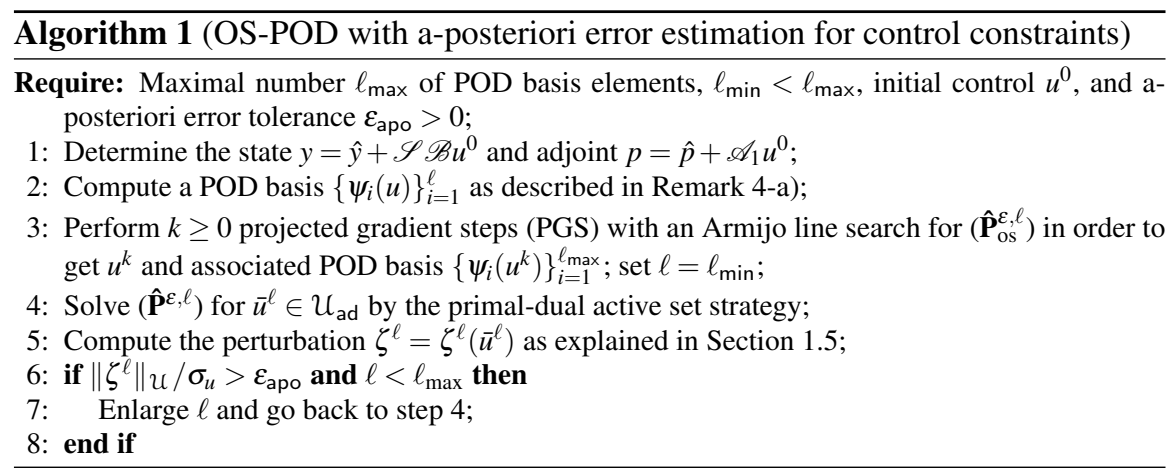

also utilize snapshots of the adjoint variable in order to compute a POD basis as described in Remark 4-a).

For the mixed constraints, this iteration turns out to not efficient enough. The gradient steps do not lead to a satisfactorily fast and accurate POD basis. Therefore, we invest more effort in the gradient steps by interacting between the projected gradient method and the primal-dual active set strategy (PDASS). In contrast to the situation of pure control constraints, we can provide basis updates based on the more accurate PDASS controls. The stratagy is explained in Algorithm 2.

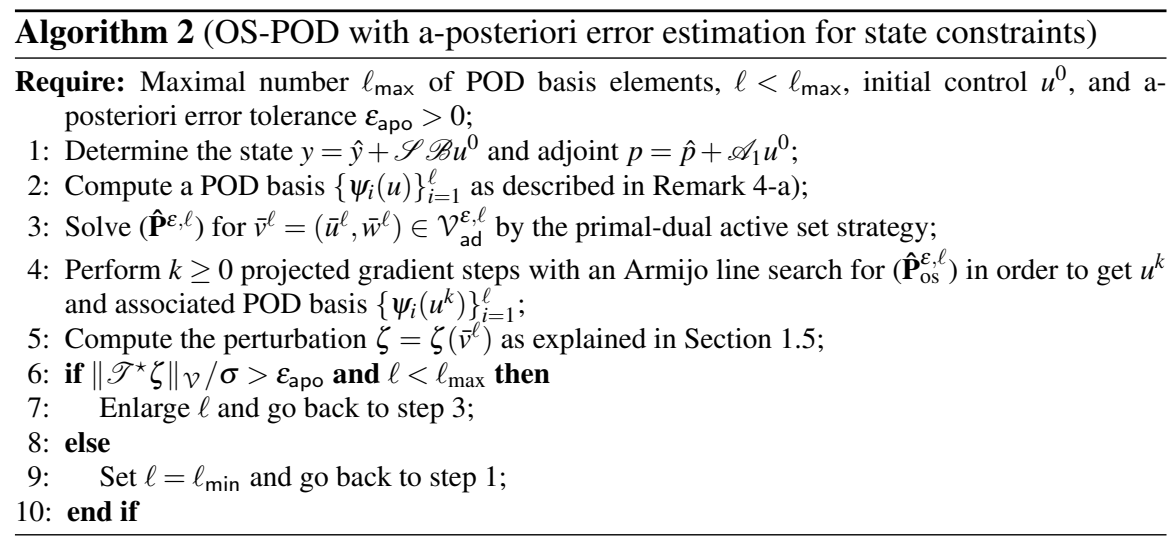




\subsection{Numerical experiments}

In this section we carry out numerical test examples illustrating the efficiency of the combination of OS-POD and a-posteriori error estimation. The evolution problems is approximated by a standard finite element (FE) method with piecewise linear finite elements for the spatial discretization. For the time integration is done by the implicit Euler method. All programs are written in MATLAB utilizing the PARTIAL DIFFERENTIAL EQUATION TOOLBOX for the FE discretization.

Run 1 (Example 1) We choose $d=2$ and consider the unit square $\Omega=(0,1) \times$ $(0,1) \subset \mathbb{R}^{2}$ as spatial domain with time interval $[0, T]=[0,1]$. The FE triangulation with maximal edge length $h=0.06$ leads to 498 degrees of freedom. For the time integration we choose an equidistant time grid $t_{j}=j \Delta t$ for $j=0, \ldots, 250$ with $\Delta t=$ 0.004. Motivated by the discretization error we set $\varepsilon_{\text {apo }}=\max \left(h^{2}, \Delta t\right)=\Delta t$. In (1.4) we choose the data $c_{p}=10, q=0.01, \tilde{f}=0$ and $y_{\circ}\left(x_{1}, x_{2}\right)=3-4\left(x_{2}-0.5\right)^{2}$; see left plot in Figure 1.1. We use $\sigma_{Q}=0, \sigma_{\Omega}=1$ and the regularization $\sigma_{u}=0.1$ in the cost
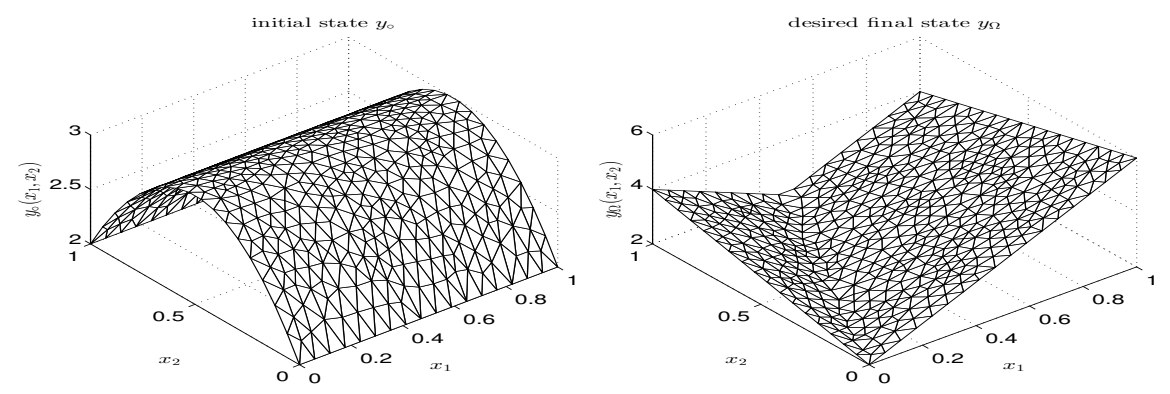

Fig. 1.1 Run 1: The initial condition $y_{\circ}$ (left) and the desired terminal state $y_{\Omega}$ (right).

function (1.3) to approximate the desired terminal state $y_{\Omega}\left(x_{1}, x_{2}\right)=2+2 \mid 2 x_{1}-$ $x_{2} \mid$; see right plot in Figure 1.1. The control constraints are chosen to be $u_{a}=0$ and $u_{b}=1$. The FE primal-dual active set strategy needs five iterations and 860.75 seconds. The optimal FE control is presented in Figure 1.2. We apply Algorithm 1 with $\ell_{\max }=40, \ell=10$ and initial control $u^{0}=0$. First we do not perform any OSPOD strategy (i.e., $k=0$ In Algorithm 1). The method stops after 110.77 seconds with $\ell=35<\ell_{\max }$ ansatz functions with $\left\|\zeta^{\ell}\right\|_{u} / \sigma_{u} \approx 0.0034<\varepsilon_{\text {apo }}$. Each solve of $\left(\hat{\mathbf{P}}^{\varepsilon, \ell}\right)$ needs four or five iterations to determine the suboptimal POD solutions. If we initialize Algorithm 1 with the optimal FE control $\bar{u}^{\mathrm{FE}}$ as initial control and perform no OS-POD strategy, only $\ell=13$ POD basis functions are required. We get $\left\|\zeta^{\ell}\right\|_{u} / \sigma_{u} \approx 0.0019<\varepsilon_{\text {apo }}$ and the CPU time is 11.48 seconds, which is ten times faster than with the initial control $u^{0}=0$. With one OS-POD gradient step, the tolerance $\varepsilon_{\text {apo }}$ is not reached with the available $\ell_{\max }=40$ basis functions. Though we make an effort in direction of the optimal control, the algorithm seems to perform even worse than with the basis corresponding to the uncontrolled state. This can be 

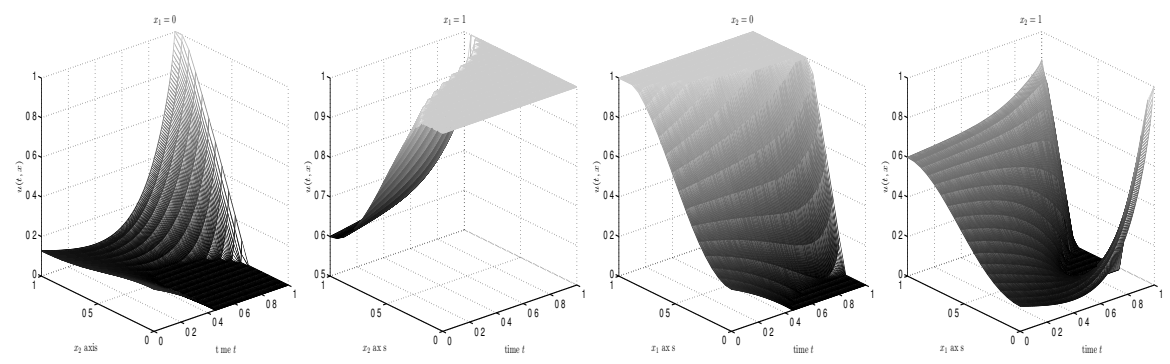

Fig. 1.2 Run 1: FE optimal control along the boundary parts $x_{1}=0, x_{1}=1, x_{2}=0$, and $x_{2}=1$.

seen in the higher control errors that cause the algorithm to run up to $\ell_{\max }=40$ ansatz functions. We can see, however, that the errors in the suboptimal state are one order smaller than without gradient steps, so the POD basis did improve after all. After $k=2$ gradient steps, the performance is considerably better: The algorithm already terminates with a ROM rank of $\ell=13$ like in the optimal case. In Table 1.1 we provide the required CPU times and final errors. Additionally regard Table 1.2

\begin{tabular}{l|cccc}
\hline & $k=0$ & $k=1$ & $k=2$ & with $\bar{u}^{\mathrm{FE}}$ \\
\hline Required $\ell$ & 35 & 40 & 13 & 13 \\
CPU time & $110.77 \mathrm{~s}$ & $147.14 \mathrm{~s}$ & $18.39 \mathrm{~s}$ & $11.48 \mathrm{~s}$ \\
\hline$\left\|\zeta^{\ell}\right\|_{u} / \sigma_{u}$ & $3.43 \cdot 10^{-3}$ & $1.14 \cdot 10^{-2}$ & $2.82 \cdot 10^{-3}$ & $1.94 \cdot 10^{-3}$ \\
$\left\|\bar{u}^{\ell}-\bar{u}^{\mathrm{FE}}\right\|_{u}$ & $3.15 \cdot 10^{-3}$ & $9.53 \cdot 10^{-3}$ & $2.62 \cdot 10^{-3}$ & $1.93 \cdot 10^{-3}$ \\
\hline
\end{tabular}

Table 1.1 Run 1: Performance of Algorithm 1.

where we compare the errors for the POD suboptimal solutions for fixed rank $\ell=15$. Here, we also provide the number of nodes that are restricted by the box constraints

\begin{tabular}{l|cccc}
\hline & $k=0$ & $k=1$ & $k=2$ & with $\bar{u}^{\mathrm{FE}}$ \\
\hline$\left\|\zeta^{\ell}\right\|_{\nu} / \sigma_{u}$ & $2.50 \cdot 10^{-2}$ & $1.45 \cdot 10^{-2}$ & $2.27 \cdot 10^{-3}$ & $1.59 \cdot 10^{-3}$ \\
$\left\|\bar{u}^{\ell}-\bar{u}^{\mathrm{FE}}\right\|_{u}$ & $2.06 \cdot 10^{-2}$ & $1.19 \cdot 10^{-2}$ & $2.07 \cdot 10^{-3}$ & $1.59 \cdot 10^{-3}$ \\
\hline different $u_{a}$ & 96 & 67 & 15 & 16 \\
different $u_{b}$ & 63 & 38 & 6 & 4 \\
\hline
\end{tabular}

Table 1.2 Run 1: Comparison of POD suboptimal solutions for $\ell=15$ and $k$ OS-POD steps.

either in the suboptimal control $\bar{u}^{15}$ or in the FE optimal control $\bar{u}^{\mathrm{FE}}$, but not in both. It tells us, how many of the restricted nodes are mistaken. This number decreases to 21 by the gradient steps. Next we are interested in the approximation of the active sets. The computations are done with 68 triangulation nodes at the boundary and 
251 time steps; that is a total amount of $68 \cdot 251=17068$ boundary nodes in the time interval $[0, T]$. The FE optimal control is restricted by $u_{a}$ at 2233 and by $u_{b}$ at 3891 nodes. In Table 1.3 we present the number of nodes where $u_{k}$ is restricted to the lower or upper bound and, in parenthesis, how many of these nodes are actually restricted correctly, i.e. equal to $\bar{u}^{\mathrm{FE}}$, what amounts to more than $99 \%$. Finally, let

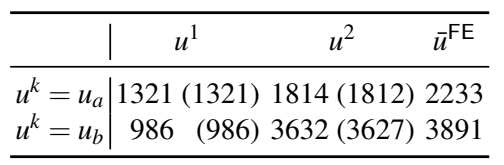

Table 1.3 Run 1: Number of active nodes. In parenthesis the number of nodes, where $u^{k}=\bar{u}^{\mathrm{FE}}=$ $u_{a}$ or $u_{k}=\bar{u}^{\mathrm{FE}}=u_{b}$, respectively.

us illustrate the changes achieved in the POD basis by the OS-POD steps. The left plot of Figure 1.3 shows how the decay of normalized eigenvalues differs depending on the used control for snapshot generation. The eigenvalues corresponding to the
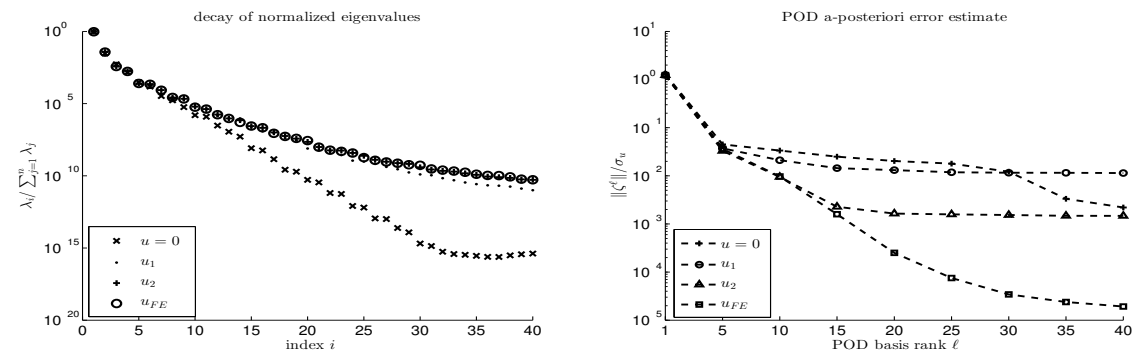

Fig. 1.3 Run 1: Comparison of eigenvalue decay for POD basis generated with $u_{k}$ after $k$ gradient steps or with $\bar{u}^{\mathrm{FE}}$ (left) and a-posteriori error for increasing $\ell$ (right).

uncontrolled state decay faster and further than those corresponding to the more or less optimally controlled state; increasing the utilized rank further than $\ell=35$ yields no more improvement. The difference caused by one gradient step is significant. A lot more basis functions contain still relevant information for the reduced order models. After the second gradient step the course is equal to the optimal situation, at least for the considered rank $\ell \leq 40$. The right plot of Figure 1.3 shows the aposteriori error for the suboptimal control. By one gradient step the control error first decreases, but then stagnates at this level. Though without any gradient step, the error is higher at the beginning, between 30 and 35 basis functions it jumps down once more and therefore the algorithm can reach the tolerance. However, the right plot shows that the absolute error in state stays far above the OS-POD results. In Figure 1.4 we compare the first four POD basis functions obtained either with $u^{0}=0, u_{2}$ or $\bar{u}^{\mathrm{FE}}$. In the first POD basis function associated with the uncontrolled equation $(u=0)$ we recognize the initial condition; see left plot of Figure 1.1. The 


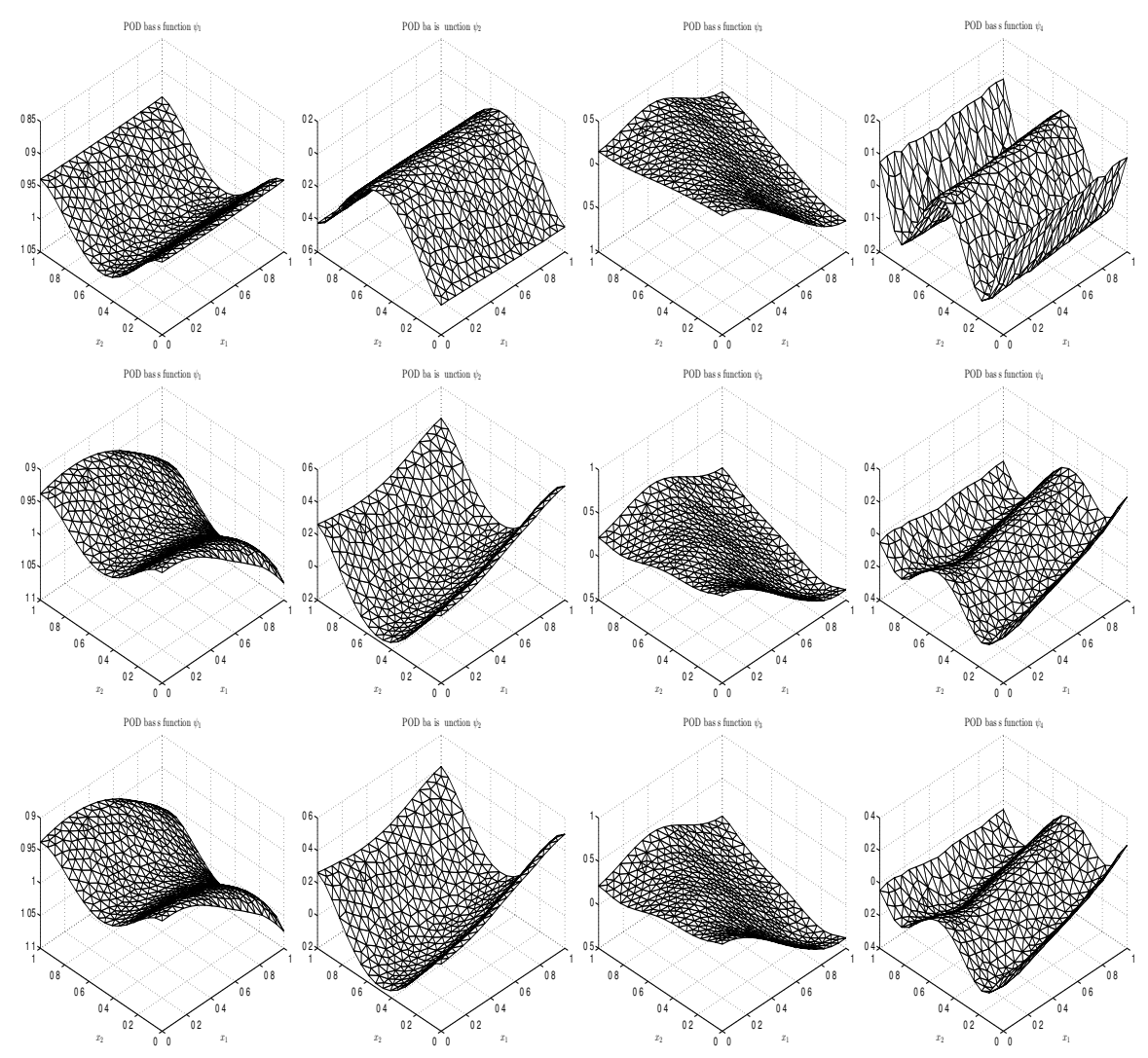

Fig. 1.4 Run 1: First four POD basis functions associated with the initial control $u^{0}=0$ (top), with the control gained after $k=2$ OS-POD steps (middle) and with the optimal FE control $\bar{u}^{\mathrm{FE}}$ (bottom).

optimal state is richer in dynamics what is reflected by a different shape of the POD basis functions. After two OS-POD steps the basis has changed significantly and at least the first four modes can hardly be distinguished from the optimal ones.

Run 2 (Example 2) As a second test, we study a distributed control problem with control and state constraints. In Example 2 we choose $d=1, v=1, \beta=-5, N_{t}=$ 400 time points in the time interval $[0,1], N_{x}=600$ grid points in the domain $\Omega=$ $[0,3], m=50$ control components and $n=800$, i.e. pontwise state constraints. For the data, we choose $f=0, y_{\circ}=\frac{1}{2} \chi_{[1.2,1.8]}$ and $y_{Q}(t, x)=\frac{1}{9}\left(6 x+6 t x-2 x^{2}\right)$ for $t<$ $1-\frac{1}{3} x, y_{Q}(t, x)=0$ elsewhere and $\sigma_{Q}=1, \sigma_{\Omega}=0, \sigma_{u}=\sigma_{w}=\varepsilon=7.5 \mathrm{e}-02$. The control and state bounds are $u_{a}=-1, u_{b}=4$ and $y_{a}=0.05, y_{b}=0.5$. Compared to the situation in Run 1 additional challenges arise here:

1. If the convection parameter $\beta$ which resembles the dispersal speed of the initial profile is dominant, a rapid decay of the singular values of the POD operator $\mathscr{R}$ 
is prevented. This results in a slower decay of the POD error $\ell \mapsto\left\|\bar{v}-\bar{v}^{\ell}\right\|_{\mathcal{V}}$, so larger POD basis ranks are required to ensure a good approximation.

2. The transport term $\beta y_{x}$ requires further considerations for the full-order solution techniques. For instance, central differences lead to a stable discretization if $v \Delta t \leq \Delta x^{2} / 2$ holds true, but nevertheless, strong oscillations of the discrete solution may occure if the conditon $|\beta \Delta x / v|<2$ is violated; see, e.g., [21]. An upwind scheme for $\beta y_{x}$ which combines forward and backward differences prevents oscillations, but is only of convergence order one.

3. By evaluation of the a-posteriori error estimator, the active set equations $\bar{u}^{\ell}=u_{a}$ and $\bar{u}^{\ell}=u_{b}$ defining the control perturbation $\zeta_{u}^{\ell}$ are fulfilled exactly by construction since $\overline{\mathfrak{v}}_{1}^{\ell}=\bar{u}^{\ell}$ holds. This is not the case for the state perturbation $\zeta_{w}^{\ell}$ : Here, a high-order solution operation is required to calculate $\overline{\mathfrak{v}}_{2}^{\ell}=\varepsilon \bar{w}^{\ell}+\mathscr{I} \mathscr{S} \mathscr{B} \bar{u}^{\ell}$ and to determine the active sets $\overline{\mathfrak{v}}_{2}^{\ell}=\hat{y}_{a}$ and $\overline{\mathfrak{v}}_{2}^{\ell}=\hat{y}_{b}$, respectively. We propose to replace the active set equalities by $\left\|\overline{\mathfrak{v}}_{2}^{\ell}-\hat{y}_{a, b}\right\|_{\mathcal{W}}<\varepsilon_{\text {acc }}$, where $\varepsilon_{\text {acc }}$ is the accuracy of the full-order model.

4. If the penalized state constraint shall resemble a pointwise pure state constraint, one may choose a fine partition $\left(\Omega_{j}\right)_{1 \leq j \leq n_{y}} \subseteq \Omega$ of $\Omega$ and $\pi_{j}(x)=\left|\Omega_{j}\right|^{-1}$ for $x \in$ $\Omega_{j}$ as well as 0 otherwise. In this case, we have $\left(\mathscr{I}_{j} y\right)(t)=\left|\Omega_{j}\right|^{-1} \int_{\Omega_{j}} y(t, x) \mathrm{d} x \approx$ $y\left(t, x_{j}\right)$. Now, choosing $\varepsilon \ll 1$ and $\sigma_{w} \gg 1$ ensures $\varepsilon w+\mathscr{I} y \approx y$ : The penalty $w$ cannot compensate strong violations of the state constraint any more. A small $\varepsilon$ leads to bad condition numbers of the optimality system matrices already for the full-order model which causes not only stability problems, but also less regular state solutions. Since the convergence of POD solutions to the full-order ones require an additional regularity of the snapshot ensemble, a good accuracy of the POD model can be expected only if additional effort is conducted for finding appropriate snapshots.

The uncontrolled FE state is plotted in the left plot of Figure 1.5. The discontinuous
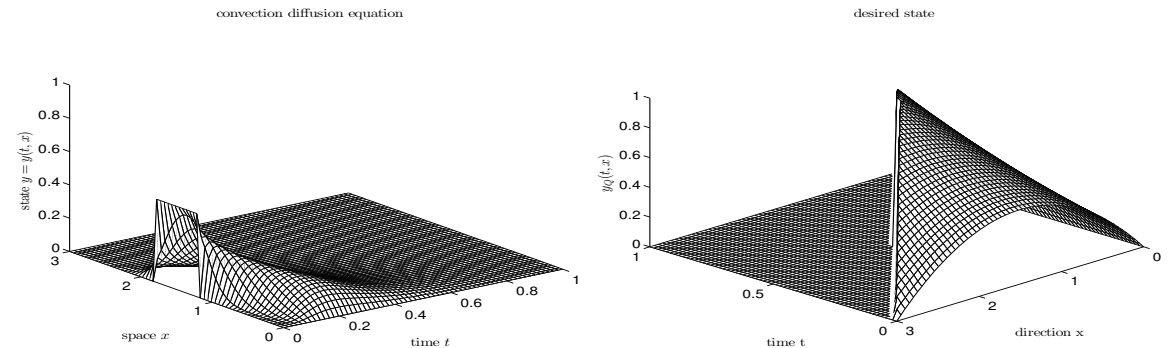

Fig. 1.5 Run 2: The uncontrolled state (left) and the desired state $y_{Q}$ (right).

desired state $y_{Q}$ is presented in the right plot of Figure 1.5. The optimal FE solution to $\left(\mathbf{P}^{\varepsilon}\right)$ is shown in Figure 1.6. The primal-dual active set strategy (PDASS) required a rather large number of iterations to converge. The complex structure of the active and inactive sets are given in Figure 1.7. In this example, 39 updates of 

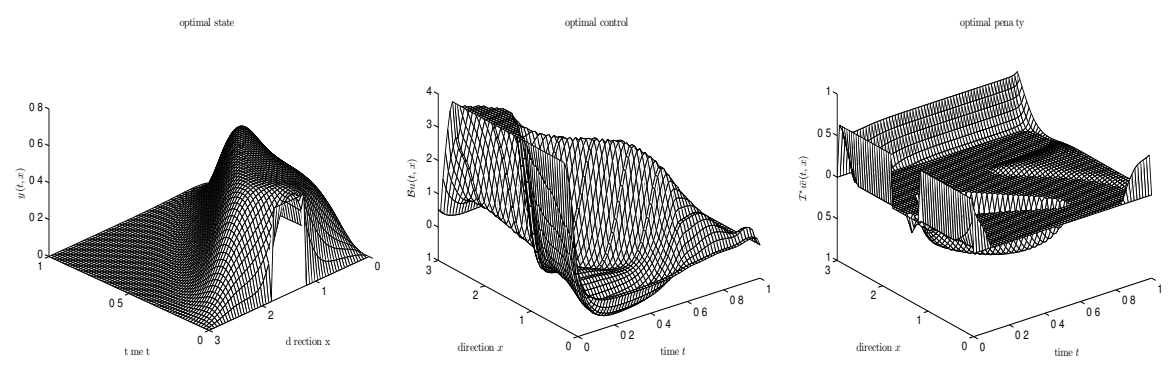

Fig. 1.6 Run 2: The optimal FE state $\bar{y}$ (left), the optimal FE control $\bar{u}$ and the optimal FE penalty $\bar{w}$ (right) to $\left(\mathbf{P}^{\varepsilon}\right)$.
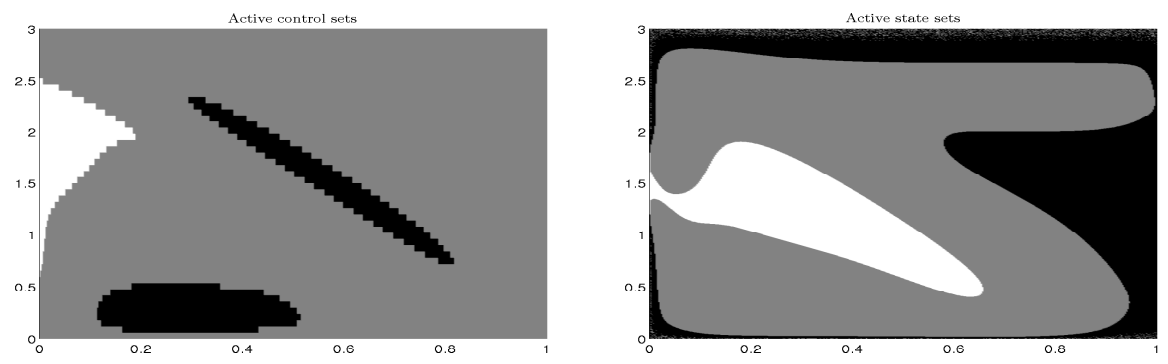

Fig. 1.7 Run 2: The active sets of the upper bounds (white) and the lower bounds (black) as well as the inactive regions (grey) for the control constraints (left) and the mixed penalty-state constraints (right).

the active sets are conducted until the iteration stops after 1217 seconds. Due to $\sigma_{u} \gg 0$ as well as the control constraints which prevent that $\bar{u}$ developes singularities and $\bar{y}$ loses regularity, the state solution is smooth. However, $\varepsilon \ll 1$ causes a plateau, where the upper state constraint $y_{b}=0.5$ is active. This dynamics which do not occur in the uncontrolled state $\hat{y}$ have to be included in appropriate snapshots to generate an accurate POD basis. Due to the strong convection, projections even on the optimal POD space spanned by the POD elements of the optimal snapshots $\bar{y}$ and $\bar{p}$ cause significant approximation errors if the POD basis rank $\ell$ is not chosen sufficiently large. Table 1.4 shows that this procedure does not lead to an adequate model error if state constraints are taken into account. The first row presents the gradient-based error indicator $\operatorname{Err}(v)=\left\|v-\mathbb{P}_{v}\left(v+d_{v}\right)\right\|_{v}$ which is our termination criterion for the projected gradient method [15]; the value almost stagnates after circa eight iterations such as the corresponding objective value $\hat{J}^{\ell}(v)$. The third line presents the POD basis ranks used for the active set strategy. We choose $\ell=\min \left\{\max \left\{v \mid \lambda_{v}>\lambda_{\min }\right\}, \ell_{\max }\right\}$ where we set $\lambda_{\min }=10^{-4}$ and $\ell_{\max }$ ensures that the model reduction effect does not vanish by using too many POD elements. We see that at least two gradient steps are required to get a sufficiently rich snapshot sample. The next row shows the number of active set updates in the reduced model. Four initializing gradient steps lead to a fast termination of this routine. However, the corresponding errors do not decay below the value 0.15 independent of the num- 


\begin{tabular}{l|cccccccc}
\hline GradSteps & 0 & 1 & 2 & 3 & 4 & 5 & 10 & 25 \\
\hline CPU time & $10.24 \mathrm{~s}$ & $22.08 \mathrm{~s}$ & $29.32 \mathrm{~s}$ & $28.64 \mathrm{~s}$ & $13.42 \mathrm{~s}$ & $15.53 \mathrm{~s}$ & $24.55 \mathrm{~s}$ & $53.46 \mathrm{~s}$ \\
\hline $\operatorname{Err}\left(v^{\nabla}\right)$ & - & 4.36 & 3.07 & 2.65 & 2.51 & 2.42 & 2.17 & 2.15 \\
$\hat{J}^{\ell}\left(v^{\nabla}\right)$ & - & 37.18 & 36.52 & 31.55 & 31.02 & 29.59 & 28.53 & 28.44 \\
$\ell\left(v^{\nabla}\right)$ & 16 & 26 & 32 & 32 & 32 & 33 & 33 & 33 \\
Iter $\left(\psi^{\ell}\right)$ & 12 & 18 & 19 & 18 & 8 & 8 & 8 & 8 \\
\hline$\left\|\bar{v}^{\ell}-\bar{v}^{\mathrm{FE}}\right\| \nu$ & $3.5 \cdot 10^{-0}$ & $1.7 \cdot 10^{-1}$ & $1.6 \cdot 10^{-1}$ & $1.6 \cdot 10^{-1}$ & $1.6 \cdot 10^{-1}$ & $1.6 \cdot 10^{-1}$ & $1.5 \cdot 10^{-1}$ & $1.6 \cdot 10^{-1}$ \\
$\left\|\mathscr{T}^{\star} \zeta^{\ell}\right\| \nu / \sigma$ & $4.4 \cdot 10^{-0}$ & $2.1 \cdot 10^{-1}$ & $1.9 \cdot 10^{-1}$ & $1.9 \cdot 10^{-1}$ & $1.8 \cdot 10^{-1}$ & $1.9 \cdot 10^{-1}$ & $1.8 \cdot 10^{-1}$ & $1.8 \cdot 10^{-1}$ \\
\hline
\end{tabular}

Table 1.4 Run 2: Error analysis for different numbers of initializing gradient steps.

ber of gradient steps or the chosen basis rank: Here, the gradient steps do not lead to a control $u^{\nabla}$ which is close enough to $\bar{u}$ to guarantee good snapshots for the POD basis. The a-posteriori error bounds $\left\|\mathscr{T}^{\star} \zeta^{\ell}\right\|_{\nu} / \sigma$ turn out to be of the same order as the errors themselves. Finally, the calculation times show that the model reduction would be very efficiently if the quality of the snapshots could be improved. Table 1.5 shows that the additional effort leads both to sufficiently small reduction errors and still very efficient calculation times: With three steps, the a-posteriori error estima-

\begin{tabular}{l|ccccc}
\hline PDASS steps & 1 & 2 & 3 & 4 & $(\bar{u}, \bar{w})$ \\
\hline CPU time & $15.63 \mathrm{~s}$ & $32.19 \mathrm{~s}$ & $51.61 \mathrm{~s}$ & $102.30 \mathrm{~s}$ & $49.32 \mathrm{~s}$ \\
\hline$\left\|\bar{v}^{\ell}-\bar{v}^{\mathrm{FE}}\right\|_{\nu}$ & $1.61 \cdot 10^{-1}$ & $3.22 \cdot 10^{-2}$ & $6.11 \cdot 10^{-3}$ & $8.49 \cdot 10^{-4}$ & $5.20 \cdot 10^{-4}$ \\
$\left\|\mathscr{T}^{\star} \zeta^{\ell}\right\| \nu / \sigma$ & $1.90 \cdot 10^{-1}$ & $3.53 \cdot 10^{-2}$ & $8.24 \cdot 10^{-3}$ & $8.61 \cdot 10^{-4}$ & $5.26 \cdot 10^{-4}$ \\
\hline
\end{tabular}

Table 1.5 Run 2: Error analysis for different numbers of gradient step/active set interactions.

tor guarantees that the reduced order model error is below the discretization error of the full order model. Solving the reduced-order problem lasts 51.61 seconds with this strategy which is just $4.24 \%$ of the full-order calculation time.

\section{References}

1. E. Arian, M. Fahl, and E.W. Sachs. Trust-region proper orthogonal decomposition for flow control. Technical Report 2000-25, ICASE, 2000.

2. P. Benner, V. Mehrmann, and D.C. Sorensen. Dimension Reduction of Large-Scale Systems. Lecture Notes in Computational Science and Engineering, vol. 45, Springer-Verlag, 2005.

3. D. Chapelle, A. Gariah, and J. Saint-Marie. Galerkin approximation with proper orthogonal decomposition: new error estimates and illustrative examples. ESAIM: Mathematical Modelling and Numerical Analysis, 46:731-757, 2012.

4. R. Dautray and J.-L. Lions. Mathematical Analysis and Numerical Methods for Science and Technology. Volume 5: Evolution Problems I. Springer, Berlin, 2000.

5. A.L. Dontchev, W.W. Hager, A.B. Poore, and B. Yang. Optimality, stability, and convergence in nonlinear control. Applied Mathematics and Optimization, 31:297-326, 1995. 
6. E. Grimm. Optimality System POD and A-Posteriori Error Analysis for Linear-Quadratic Optimal Control Problems. Master thesis, University of Konstanz, 2013. https://kops.unikonstanz.de/handle/123456789/27761

7. M. Gubisch and S. Volkwein. Proper orthogonal decomposition for linear-quadratic optimal control. Submitted, 2013. http://kops.uni-konstanz.de/handle/123456789/25037

8. M. Gubisch and S. Volkwein. POD a-posteriori error analysis for optimal control problems with mixed control-state constraints. Computational Optimization and Applications, 58:619644, 2014.

9. M. Hintermüller, K. Ito, and K. Kunisch. The primal-dual active set strategy as a semismooth Newton method. SIAM Journal on Optimization, 13:865-888, 2003.

10. M. Hintermüller, I. Kopacka, and S. Volkwein. Mesh-independence and preconditioning for solving control problems with mixed control-state constraints. ESAIM: Control, Optimisation and Calculus of Variations, 15:626-652, 2009.

11. P. Holmes, J.L. Lumley, G. Berkooz, and C.W. Rowley. Turbulence, Coherent Structures, Dynamical Systems and Symmetry. Cambridge Monographs on Mechanics, Cambridge University Press, second edition, 2012.

12. M. Hinze, R. Pinnau, M. Ulbrich, and S. Ulbrich. Optimization with PDE Constraints. Springer-Verlag, Berlin, 2009.

13. M. Hinze and S. Volkwein. Proper orthogonal decomposition surrogate models for nonlinear dynamical systems: error estimates and suboptimal control. Chapter 10 of [2].

14. M. Hinze and S. Volkwein. Error estimates for abstract linear-quadratic optimal control problems using proper orthogonal decomposition. Computational Optimization and Applications, 39:319-345, 2008.

15. C.T. Kelley. Iterative Methods for Optimization. SIAM Frontiers in Applied Mathematics, Philadelphia, 1999.

16. K. Kunisch and S. Volkwein. Galerkin proper orthogonal decomposition methods for a general equation in fluid dynamics. SIAM Journal on Numerical Analysis, 40:492-515, 2002.

17. K. Kunisch und S. Volkwein. Proper orthogonal decomposition for optimality systems. ESAIM: Mathematical Modelling and Numerical Analysis, 42:1-23, 2008.

18. M. Müller. Uniform Convergence of the POD Method and Applications to Optimal Control. Ph.D thesis, University of Graz, 2011.

19. W.H.A. Schilders, H.A. van der Vorst, and J. Rommes. Model Order Reduction: Theory, Research Aspects and Applications. Mathematics in Industry, vol. 13, Springer, 2008.

20. J.R. Singler. New POD expressions, error bounds, and asymptotic results for reduced order models of parabolic PDEs. SIAM Journal on Numerical Analysis, 52:852-876, 2014.

21. J. Strikwerda. Finite Difference Schemes and Partial Differential Equations. SIAM, Philadelphia, 2004.

22. A. Studinger and S. Volkwein. Numerical analysis of POD a-posteriori error estimation for optimal control. International Series of Numerical Mathematics, 164:137-158, 2013

23. F. Tröltzsch. Regular Lagrange multipliers for control problems with mixed pointwise controlstate constraints. SIAM Journal on Optimization, 22:616-634, 2005.

24. F. Tröltzsch. Optimal Control of Partial Differential Equations. Theory, Methods and Applications. American Mathematical Society, Providence, volume 112, 2010.

25. F. Tröltzsch and S. Volkwein. POD a-posteriori error estimates for linear-quadratic optimal control problems. Computational Optimization and Applications, 44:83-115, 2009.

26. S. Volkwein. Optimality system POD and a-posteriori error analysis for linear-quadratic problems. Control and Cybernetics, 40:1109-1125, 2011. 